International Journal of Modern Physics D

(C) World Scientific Publishing Company

\title{
DARK ENERGY EQUATION OF STATE AND COSMIC TOPOLOGY
}

\author{
SANDRO D. P. VITENTI, MARIANA P. LIMA AND MARCELO J. REBOUÇAS \\ Centro Brasileiro de Pesquisas Físicas, Rua Dr. Xavier Sigaud 150 \\ 22290-180 Rio de Janeiro - RJ, Brasil \\ vitenti@cbpf.br, penna@cbpf.br, reboucas@cbpf.br
}

Received Day Month Year

Revised Day Month Year

Communicated by Managing Editor

\begin{abstract}
The immediate observational consequence of a non-trivial spatial topology of the Universe is that an observer could potentially detect multiple images of radiating sources. In particular, a non-trivial topology will generate pairs of correlated circles of temperature fluctuations in the anisotropies maps of the cosmic microwave background (CMB), the so-called circles-in-the-sky. In this way, a detectable non-trivial spatial topology may be seen as an observable attribute, which can be probed through the circles-in-the-sky for all locally homogeneous and isotropic universes with no assumptions on the cosmological dark energy (DE) equation of state (EOS) parameters. We show that the knowledge of the spatial topology through the circles-in-the-sky offers an effective way of reducing the degeneracies in the DE EOS parameters. We concretely illustrate the topological role by assuming a Poincaré dodecahedral space topology and reanalyzing the constraints on the parameters of a specific EOS which arise from the supernovae type Ia, baryon acoustic oscillations and the CMB plus the statistical topological contribution.
\end{abstract}

Keywords: Dark energy equation of state parameters; cosmic topology.

\section{Introduction}

In the standard cosmology, the Universe is modelled by a space-time manifold $\mathcal{M}_{4}=$ $\mathbb{R} \times M_{3}$ endowed with a locally (spatially) homogeneous and isotropic RobertsonWalker metric

$$
d s^{2}=-c^{2} d t^{2}+a^{2}(t)\left[d \chi^{2}+S_{k}^{2}(\chi)\left(d \theta^{2}+\sin ^{2} \theta d \phi^{2}\right)\right],
$$

where $a(t)$ is the cosmological scale factor, and $S_{k}(\chi)=(\chi, \sin \chi, \sinh \chi)$ depending on the sign of the constant curvature $(k=0,1,-1)$ of the spatial sections $M_{3}$. The 3 -space $M_{3}$ is usually taken to be one of the simply-connected manilfolds: Euclidean $\mathbb{R}^{3}$, spherical $\mathbb{S}^{3}$, or hyperbolic $\mathbb{H}^{3}$. However, it is known that the great majority of such constant curvature 3-spaces are multiply-connected quotient manifolds of the form $\mathbb{R}^{3} / \Gamma, \mathbb{S}^{3} / \Gamma$, and $\mathbb{H}^{3} / \Gamma$, where $\Gamma$ is a fixed-point free group of isometries of the corresponding covering space. On the other hand, given that the connectedness of the spatial sections $M_{3}$ has not been determined by cosmological observations, and 
since geometry does not fix the topology, our 3-dimensional space may be one of these possible multiply-connected quotient manifolds (for reviews on this issue see, e.g., the Refs. (1).

The immediate observational consequence of a detectable nontrivial topology 2 of $M_{3}$ is the existence of the circles-in-the-sky, i.e., pairs of matching circles will be imprinted on the cosmic microwave background (CMB) anisotropy sky maps. $\stackrel{3}{[}$ Hence, to observationally probe a putative nontrivial topology of $M_{3}$, one should examine the CMB maps in order to extract the pairs of correlated circles and determine the spatial topology.

In this regard, in a few recent works 4 in the context of $\Lambda$ cold dark matter $(\Lambda \mathrm{CDM})$ model, it has been shown that the knowledge of the spatial topology through the circles-in-the-sky offers an effective way of setting constraints on the density parameters associated with matter $\left(\Omega_{m}\right)$ and dark energy $\left(\Omega_{\Lambda}\right)$. In other words, it has been shown in Refs. 4 that specific circles-in-the-sky detectable topology of the spatial section of the Universe can be used to reduce the degeneracies in the density parameters plane $\Omega_{m}-\Omega_{\Lambda}$, which arise from statistical analyses with data from current observations (see also the related Refs. 5).

The question as to whether the detection of a non-trivial cosmic topology can also be used to set constraints on the equation of state parameters naturally arises here. In this paper we address this question by examining to what extent a nontrivial spatial topology can be used to place further constraints on the dark energy (DE) equation of state parameter $w_{x}$. To this end, we shall assume the Poincaré dodecahedral space as the circles-in-the-sky observable spatial topology, and reanalyze the current constraints on a two-parameter DE equation of state (EOS) of Ref. 6, which arise from the Type Ia supernovae (SNe Ia) data from the Legacy sample 7 along with the baryon acoustic oscillations (BAO) peak in the large-scale correlation function of the Sloan Digital Sky Survey (SDSS), 8 and CMB shift parameter. 9

\section{Poincaré Dodecahedral Space}

Combining the first-year CMB with other astronomical data, the WMAP team 10 reported the estimated value of the total density $\Omega_{\mathrm{tot}}=1.02 \pm 0.02(1 \sigma$ level $)$, which includes a positively-curved universe as a possibility. This reported sign for the curvature density $\left(\Omega_{k}=1-\Omega_{\text {tot }} \leq 0\right)$ has been reinforced by the combination of WMAP three year CMB measurements with other observational data sets, cf. Table 12 of Ref. 11, wherein six different values for the $\Omega_{\text {tot }}$ ranging from a very nearly flat $\left(\Omega_{\text {tot }}=1.005_{-0.0068}^{+0.0060}\right)$ to positively curved $\left(\Omega_{\text {tot }}=1.023 \pm 0.014\right)$ depending on the combination of data sets used to resolve the geometrical degeneracy.

In this work we assume the positively-curved Poincaré dodecahedral space (PDS) as the circles-in-the-sky detectable topology of the spatial sections of the Universe

a This topology is consistent with the observed total density, and accounts for both the suppression of power of the low multipoles of the first and three year WMAP data $10 \mid 11$ and the temperature two-point correlation function (see Refs. 12, 14). 
The fundamental polyhedron (FP) of the PDS topology is a regular spherical dodecahedron that tiles the covering space $\mathbb{S}^{3}$ into 120 identical cells. The radius $r_{\text {inj }}$ (called the injectivity radius) of the smallest sphere 'inscribable' in the FP is $\pi / 10$. The PDS is globally homogeneous, and a detectable PDS topology $\left(\chi_{\mathrm{lss}}>r_{\mathrm{inj}}\right.$, cf. Ref. 2) gives rise to six pairs of antipodal circles-in-the-sky, one of which is shown in Fig 1 .

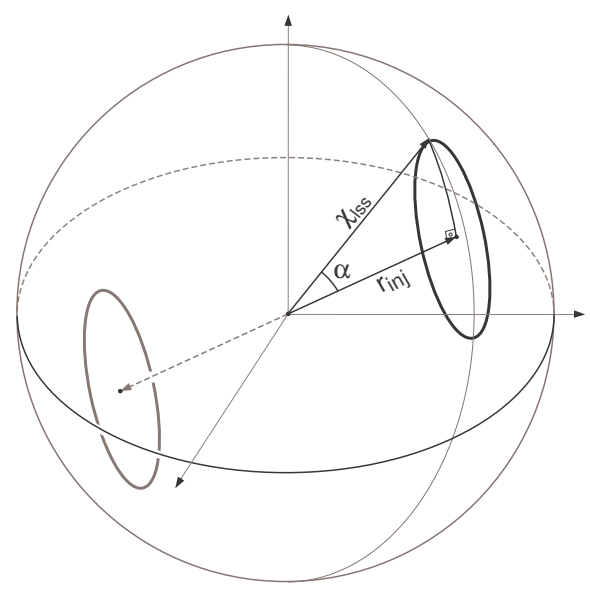

Fig. 1. A schematic illustration of a pair of antipodal matching circles on the sphere of last scattering. The relation between the angular radius $\alpha$ of the circles and the angular sides $r_{\mathrm{inj}}$ and $\chi_{\mathrm{lss}}$ is given by Eq.(2).

Now, a straightforward use of a Napier's rule to the right-angled spherical triangle furnishes a relation between the angular radius $\alpha$ and the angular sides $r_{\text {inj }}$ and $\chi_{\mathrm{lss}}$ of the last scattering sphere, namely

$$
\cos \alpha=\frac{\tan r_{\mathrm{inj}}}{\tan \chi_{\mathrm{lss}}}
$$

which can be solved for $\chi_{\text {lss }}$ to give

$$
\chi_{\mathrm{lss}}=\tan ^{-1}\left[\frac{\tan r_{\mathrm{inj}}}{\cos \alpha}\right] .
$$

This equation shows that for a given topology, the measurement of a value $\alpha$ for a circle-in-the-sky radius gives the distance $\chi_{\text {lss }}$ to the last scattering surface in units of the curvature radius today, $a_{0}=a\left(t_{0}\right)=\left(H_{0} \sqrt{\left|1-\Omega_{\mathrm{tot}}\right|}\right)^{-1}$. Given a detection of a circle-in-the-sky, one can further constrain the EOS parameters, as we shall show in Sec. 4. by comparing $\chi_{\text {lss }}$ given by Eq. (3) with that predicted by a model with specific EOS parametrization. 


\section{Dark Energy Parametrization}

In the light of current observations, we assume the Friedmann-Lemaitre-RobertsonWalker (FLRW) framework in which the current matter content of the Universe is well approximated by a dust of density $\rho_{m}$ (baryonic plus dark matter) along with a dark energy perfect fluid component of density $\rho_{x}$ and pressure $p_{x}$. The Friedmann equation is then given by

$$
H^{2}=\frac{8 \pi G}{3 c^{2}}\left(\rho_{m}+\rho_{x}\right)-\frac{k c^{2}}{a^{2}},
$$

where $H=\dot{a} / a$ is the Hubble parameter, overdot stands for derivative with respect to time $t, G$ is Newton's constant, and $c$ is the speed of light.

If one further assumes that these fluid components do not interact, the energy conservation equations reduce to

$$
\dot{\rho}_{i}+3 H\left(\rho_{i}+p_{i}\right)=0,
$$

for each component $i=m, x$. This equation can be rewritten in terms of the redshift $1+z=a_{0} / a$ and the equation of state parameters $w_{i}=p_{i} / \rho_{i}$ in the form

$$
\frac{d \rho_{i}}{d z}=\frac{3 \rho_{i}\left(1+w_{i}\right)}{1+z}
$$

and has an integral solution

$$
\rho_{i}(z)=\rho_{i}(0) \exp \left[\int_{0}^{z} \frac{3\left(1+w_{i}\right)}{1+z^{\prime}} d z^{\prime}\right] .
$$

In this way, the Friedmann equation (44) can now be rewritten in terms of the redshift $z$ and the dimensionless density variables $\Omega_{i}=\rho_{i}(0) / \rho_{\text {crit }}(0)$, where $\rho_{\text {crit }}(z)=3 c^{2} H^{2}(z) /(8 \pi G)$, in the form

$$
E^{2}(z)=\Omega_{m}(1+z)^{3}+\Omega_{k}(1+z)^{2}+\Omega_{x} f(z),
$$

where

$$
f(z)=\exp \left[\int_{0}^{z} \frac{3\left(1+w_{x}\left(z^{\prime}\right)\right)}{1+z^{\prime}} d z^{\prime}\right]
$$

and where $E(z)=H(z) / H_{0}$ is the dimensionless Hubble function and $\Omega_{k}=$ $-k c^{2} /\left(a_{0}^{2} H_{0}^{2}\right)$ is the density curvature parameter.

In this work we shall consider a model that is defined by Eq. (8) and Eq. (9) along with the Jassal-Bagla-Padmanabhan $\underline{6}$ (JBP) dark energy parametrization

$$
w_{x}(z)=w_{0}+\frac{w_{1} z}{(1+z)^{2}},
$$

which combined with Eqs. (8) and (9) give

$$
E^{2}(z)=\Omega_{m}(1+z)^{3}+\Omega_{k}(1+z)^{2}+\Omega_{x}(1+z)^{3\left(1+w_{0}\right)} \exp \left[\frac{3 w_{1} z^{2}}{2(1+z)^{2}}\right] .
$$

We note that for $z=0$ one has the constraint equation $\Omega_{x}=1-\Omega_{k}-\Omega_{m}$. 
From Eq. (3) it is clear that to study the constraints on the parameters of the JPB model we need the theoretical value of $\chi_{\text {lss }}$ predicted by this model, which is given by

$$
\chi_{\mathrm{lss}}=\sqrt{\left|\Omega_{k}\right|} \frac{d_{\mathrm{c}}\left(z_{\mathrm{lss}}\right)}{d_{H}}=\sqrt{\left|\Omega_{k}\right|} \int_{0}^{z_{l s s}} \frac{d z^{\prime}}{E\left(z^{\prime}\right)}
$$

where $d_{\mathrm{c}}(z)=d_{H} \int_{0}^{z} d z^{\prime} / E\left(z^{\prime}\right)$ is the comoving distance, $d_{H}=c / H_{0}$ is the Hubble distance today, and $z_{\mathrm{lss}}$ is the redshift of the last scattering surface.

\section{Observational Constraints}

To illustrate the role played by the PDS topology in constraining the EOS parameters of the model discussed in the previous section, we have reanalyzed, with and without the topological contribution, the constraints on the parameters that arise from Legacy sample 7 of SNe Ia along with the baryon acoustic oscillations (BAO) peak position in the galaxy power spectrum,, 8 and CMB shift parameter .9

For the sake of brevity, we shall not discuss here the likelihoods for the $\mathrm{SNe}$ Ia, BAO and CMB data, and simply refer to them as $L_{\mathrm{SN}}(\vec{P}), L_{\mathrm{BAO}}(\vec{P})$ and $L_{\mathrm{CMB}}(\vec{P})$ with $\vec{P} \equiv\left(\Omega_{m}, \Omega_{k}, w_{0}, w_{1}\right)$. Further details on these likelihoods can be found in a number of papers as, for example, in Refs. 4b and 5 b. In this way, we focus on the likelihood of topological origin, which is included in the statistical analysis as a gaussian prior as follows. The measurement of a circle-in-the-sky of radius $\alpha$ in CMB map involves unavoidably observational uncertainty $\sigma_{\alpha}$. These observational pieces of topological information can be included in the statistical analyses by using that $r_{\mathrm{inj}}=\pi / 10$ (for PDS topology) along with Eq. (3) to have $\chi_{\mathrm{lss}}$ and $\sigma_{\chi_{\mathrm{lss}}}$ (through the usual error propagation formula). In this way, a detection of a PDS circles-in-the-sky would provide an observational value of the radius of the last scattering surface and the associated uncertainty, i.e. $\chi_{\mathrm{lss}} \pm \sigma_{\chi_{\mathrm{lss}}}$, which we compare with the theoretical values predicted by the JPB model by assuming a gaussian distribution with the mean value given by Eqs. (11) and (12). Thus, the likelihood associated to the topology is given by

$$
L_{\mathrm{Top}}(\vec{P}) \propto \exp \left\{-\frac{1}{2} \frac{\left[\chi_{\mathrm{lss}}-\sqrt{\left|\Omega_{k}\right|} d_{\mathrm{c}}\left(z_{\mathrm{lss}}\right) d_{H}^{-1}\right]^{2}}{\left(\sigma_{\chi_{\mathrm{lss}}}\right)^{2}}\right\},
$$

whose dependence on the model parameters $\vec{P}=\left(\Omega_{m}, \Omega_{k}, w_{0}, w_{1}\right)$ clearly comes explicitly from $\Omega_{k}$ and from the comoving distance $d_{\mathrm{c}}\left(z_{\mathrm{lss}}\right)$.

\section{Results and Conclusions}

In order to make apparent the role of the topological term $L_{\text {Top }}$ in constraining the equation of state parameters, we have performed the joint analysis maximizing the 


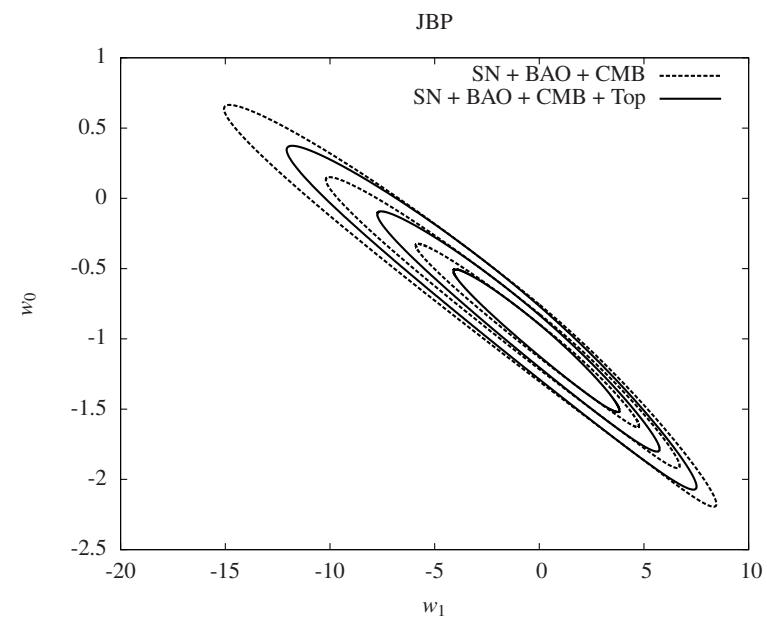

Fig. 2. Confidence regions for $1 \sigma-3 \sigma$ in the $w_{1}-w_{0}$ plane for JBP parametrization. The dashed and full lines indicate the confidence regions without and with the topological term $L_{\text {Top }}(\vec{P})$. Clearly the PDS topology reduces the degeneracies in the EOS parameters $w_{0}$ and $w_{1}$ which arise from the SNe Ia, BAO and CMB.

likelihoods with and without the topological term, namely,

$$
\begin{aligned}
& L_{1}(\vec{P})=L_{\mathrm{SN}}(\vec{P}) L_{\mathrm{BAO}}(\vec{P}) L_{\mathrm{CMB}}(\vec{P}) L_{\mathrm{Top}}(\vec{P}), \\
& L_{2}(\vec{P})=L_{\mathrm{SN}}(\vec{P}) L_{\mathrm{BAO}}(\vec{P}) L_{\mathrm{CMB}}(\vec{P}),
\end{aligned}
$$

where $L_{\text {Top }}(\vec{P})$ is given by a circle-in-the-sky radius along with an unavoidable observational uncertainty in its measurements, which we take to $\alpha=20^{\circ} \pm 4^{\circ}$. This value for the radius was not covered by the search for almost antipodal correlated circles (with radii larger than $\alpha=25^{\circ}$ ) made in Ref. 16, in which no circles were found $\mathrm{b}$ We also used a conservative error of $20 \%$ to account for the possible uncertainties in the measurement of the circle radius.

Figure 2 shows $1 \sigma-3 \sigma$ confidence regions of the JBP parameter $w_{1}-w_{0}$ plane calculated by using the above likelihoods $L_{1}(\vec{P})$ (with topology) and $L_{2}(\vec{P})$ (with no topology) and marginalizing over the remaining parameters $\Omega_{m}$ and $\Omega_{k}$. It is clear from this figure that a circles-in-the-sky detection of the PDS topology reduces appreciably the parametric regions allowed by Legacy SNe Ia sample combined with the BAO peak position in the galaxy power spectrum, and CMB shift parameter. In Tab. 1 we explicitly quantify these constraints by collecting together the $3 \sigma$ lower

\footnotetext{
${ }^{\mathrm{b}}$ We note that another statistical technique for probing below the limit of $20^{\circ}$ for a directed search for a specific topology was introduced in Ref. 17. By using this combined search procedure with the knowledge of four or more circle pairs, for example, one might be able to detect circles with radii as small as $5^{\circ}$.
} 
and upper bounds of the parameters $w_{0}$ and $w_{1}$ which were calculated without and with the topological statistical term. Table 1 makes apparent that the allowed intervals for $w_{0}$ and $w_{1}$ are reduced, respectively, of $\sim 14.4 \%$ and $\sim 16.7 \%$ by the topological term.

Table 1. The lower and upper $3 \sigma$ limits of in the $w_{0}-w_{1}$ plane without and with $L_{\text {Top }}$.

\begin{tabular}{lcc}
\hline Model & $w_{0}$ & $w_{1}$ \\
\hline JBP & $(-2.194,0.665)$ & $(-15.059,8.457)$ \\
JBP+PDS & $(-2.073,0.374)$ & $(-12.069,7.510)$ \\
\hline
\end{tabular}

To complete this study we show in Figure 3 the results of $1 \sigma-3 \sigma$ statistical analysis for $\Omega_{k}-w_{0}$ and $\Omega_{k}-w_{1}$ planes (again marginalizing over the other parameters). This figure makes apparent that the main role of the topology in these parameter planes is to reduce considerably the degeneracy on $\Omega_{k}$ allowed by the above-mentioned observational data, in agreement with earlier related results obtained in different contexts. ${ }^{5}$ We also note that the $w_{0}$ and $w_{1} 3 \sigma$ limits are the same on both Fig. 2 and Fig. 3. Finally, we note that it can be shown that a suitable Gaussian prior on the curvature density parameter $\Omega_{k}$ can mimic the role of the topology in such statistical analyses.

To conclude, we note that although we have illustrated the degree to which a circle-in-the-sky topology detection reduces the degeneracies in the DE EOS parameters by using $\alpha=20^{\circ} \pm 4^{\circ}$, our general results should not change appreciably for smaller radius and uncertainties, and its general features should hold for other two-parameters EOS.
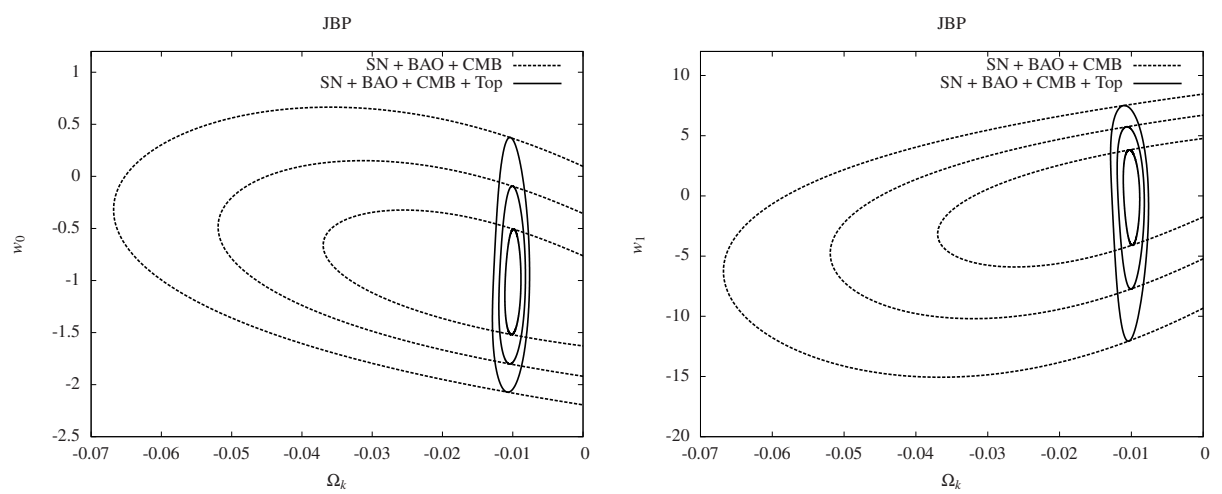

Fig. 3. Confidence regions in $1 \sigma-3 \sigma$ of $\left(\Omega_{k}, w_{0}\right)$ and $\left(\Omega_{k}, w_{1}\right)$ for JBP parametrization. The main effect of the topological prior is to constrain the value of $\Omega_{k}$. 


\section{Acknowledgments}

M. J. R. acknowledges the support of FAPERJ under a CNE E-26/101.556/2010 grant. This work was also supported by Conselho Nacional de Desenvolvimento Científico e Tecnológico (CNPq) - Brasil, under grant No. 472436/2007-4. M.J.R., S.V. and M.P.L. thank CNPq for the grants under which this work was carried out. We are also grateful to A.F.F. Teixeira for indicating misprints and omissions.

\section{References}

1. G. F. R. Ellis, Gen. Relativ. Gravitation 2 (1971) 7; M. Lachièze-Rey and J. P. Luminet, Phys. Rep. 254 (1995) 135, arXiv:gr-qc/9605010 G. D. Starkman, Class. Quantum Grav. 15 (1998) 2529; J. Levin, Phys. Rep. 365 (2002) 251, arXiv:gr-qc/0108043; M. J. Rebouças and G. I. Gomero, Braz. J. Phys. 34 (2004) 1358, arXiv:astro-ph/0402324; M. J. Rebouças, A Brief Introduction to Cosmic Topology, in Proc. XIth Brazilian School of Cosmology and Gravitation, eds. M. Novello and S. E. Perez Bergliaffa (Americal Institute of Physics, Melville, New York, 2005) AIP Conference Proceedings vol. 782, p 188. Also arXiv:astro-ph/0504365.

2. G. I. Gomero, M. J. Rebouças and R. Tavakol, Class. Quantum Grav. 18 (2001) 4461, arXiv:gr-qc/0105002 G. I. Gomero, M. J. Rebouças and R. Tavakol, Class. Quantum Grav. 18 (2001) L145, arXiv:gr-qc/0106044; G. I. Gomero, M. J. Rebouças and R. Tavakol, Int. J. Mod. Phys. A 17 (2002) 4261, arXiv:gr-qc/0210016 J. R. Weeks, Mod. Phys. Lett. A 18 (2003) 2099, arXiv:astro-ph/0212006; G. I. Gomero and M. J. Rebouças, Phys. Lett. A 311 (2003) 319, arXiv:gr-qc/0202094; B. Mota, M. J. Rebouças and R. Tavakol, Class. Quantum Grav. 20 (2003) 4837, arXiv:gr-qc/0308063 B. Mota, G. I. Gomero, M. J. Rebouças and R. Tavakol, Class. Quantum Grav. 21 (2004) 3361, astro-ph/0309371 B. Mota, M. J. Rebouças and R. Tavakol, Int. J. Mod. Phys. A 20 (2005) 2415, astro-ph/0503683; B. Mota, M. J. Rebouças and R. Tavakol, Phys. Rev. $D 78$ (2008) 083521, arXiv:0808.1572 1 [astro-ph]; B. Mota, M. J. Rebouças and R. Tavakol, Phys. Rev. D 81 (2010) 103516, arXiv:1002.0834 [astro-ph.CO]; B. Mota, M. J. Rebouças and R. Tavakol, arXiv:1007.3466 [astro-ph.CO].

3. N. Cornish, D. Spergel and G. Starkman, Class. Quantum Grav. 15 (1998) 2657, arXiv:astro-ph/9801212 M. O. Calvão, G. I. Gomero, B. Mota and M. J. Rebouças, Class. Quantum Grav. 22 (2005) 1991, arXiv:astro-ph/0404536

4. M. J. Rebouças, J. S. Alcaniz, B. Mota and M. Makler, Astron. Astrophys. 452 (2006) 803, arXiv:astro-ph/0511007; M. J. Rebouças and J. S. Alcaniz, Mon. Not. R. Astron. Soc. 369 (2006) 1693, arXiv:astro-ph/0603206. M. J. Rebouças and J. S. Alcaniz, Braz. J. Phys. 35 (2005) 1062, arXiv:astro-ph/0604087, M. J. Rebouças, Int. J. Mod. Phys. D 16 (2007) 207, arXiv:astro-ph/0605214; M. J. Rebouças, (2007), arXiv:astro-ph/0702642

5. M. C. Bento, O. Bertolami, M. J. Rebouças and P. T. Silva, Phys. Rev. D 73 (2006) 043504, arXiv:gr-qc/0512158 M. C. Bento, O. Bertolami, M. J. Rebouças and N. M. C. Santos, Phys. Rev. D 73 (2006) 103521; M. J. Rebouças, arXiv:astro-ph/0702428; M. J. Rebouças, arXiv:astro-ph/0702642 v1; M. J. Rebouças, Int. J. Mod. Phys.A 24 (2009) 1625, arXiv:0902.0613 [astro-ph.CO]; M. J. Rebouças and A. F. F. Teixeira, arXiv:1005.0097 [astro-ph.CO].

6. H. K. Jassal, J. S. Bagla and T. Padmanabhan, Mon. Not. R. Astron. Soc. 356 (2005)

L11-L16, astro-ph/0404378

7. P. Astier et al., Astron. Astrophys. 447 (2006) 31, arXiv:astro-ph/0510447

8. D. J. Eisenstein et al., Astrophys.J. 633 (2005) 560, arXiv:astro-ph/0501171. 
9. J. R. Bond, G. Efstathiou and M. Tegmark, Mon. Not. R. Astron. Soc. 291 (1997) L33, arXiv:astro-ph/9702100.

10. D. N. Spergel et al., Astrophys. J. Suppl. Ser. 148 (2003) 175, arXiv:astro-ph/0302209

11. D. N. Spergel et al., Astrophys. J. Suppl. Ser. 170 (2007) 377, arXiv:astro-ph/0603449.

12. J. P. Luminet, J. Weeks, A. Riazuelo, R. Lehoucq and J. P. Uzan, Nature 425 (2003) 593, arXiv:astro-ph/0310253

13. R. Aurich, S. Lustig and F. Steiner, Class. Quantum Grav. 22 (2005) 2061, arXiv:astro-ph/0412569

14. R. Aurich, S. Lustig and F. Steiner, Class. Quantum Grav. 22 (2005) 3443, arXiv:astro-ph/0504656

15. Y. Wang and P. Mukherjee, Astrophys. J. 650 (2006) 1, arXiv:astro-ph/0604051

16. N. J. Cornish, D. N. Spergel, G. D. Starkman and E. Komatsu, Phys. Rev. Lett. 92, (2004) 201302, arXiv:astro-ph/0310233.

17. J. S. Key, N. J. Cornish, D. N. Spergel and G. D. Starkman, Phys. Rev. D 75 (2007) 084034, arXiv:astro-ph/0604616v1. 